\title{
An Extensible Query Language for Content Based Image Retrieval
}

\author{
Raoul Pascal Pein ${ }^{*, 1,2}$, Zhongyu Lu ${ }^{1}$ and Wolfgang Renz ${ }^{2}$ \\ ${ }^{I}$ Department of Informatics, School of Computing and Engineering, University of Huddersfield, Queensgate, \\ Huddersfield HDI $3 D H, U K$ \\ ${ }^{2}$ Multimedia Systems Laboratory (MMLab), Faculty of Engineering and Computer Science, Hamburg University of \\ Applied Sciences, Berliner Tor 7, 20099 Hamburg, Germany
}

\begin{abstract}
One of the most important bits of every search engine is the query interface. Complex interfaces may cause users to struggle in learning the handling. An example is the query language SQL. It is really powerful, but usually remains hidden to the common user. On the other hand the usage of current languages for Internet search engines is very simple and straightforward. Even beginners are able to find relevant documents.

This paper presents a hybrid query language suitable for both image and text retrieval. It is very similar to those of a full text search engine but also includes some extensions required for content based image retrieval. The language is extensible to cover arbitrary feature vectors and handle fuzzy queries.
\end{abstract}

\section{INTRODUCTION}

After several years of research the idea of content based image retrieval (CBIR) (Eakins JISCTAP 1999) [1] (Renz OTDGI 2000) [2] is still not established in daily life. Currently most effort in CBIR is put into closing the semantic gap between simple visual features and the real image semantics. The work done in this area is very important to allow untrained users to work with image retrieval systems. A survey about such systems is available from Liu (Liu PR 2007) [3]. None of the systems analysed there is really capable of closing the gap completely. Either the solutions are far too specific or require much human attention. The most important open tasks identified are "query-language design, integration of image retrieval with database management system, high-dimensional image feature indexing" and "integration of salient low-level feature extraction, effective learning of high-level semantics, friendly user interface, and efficient indexing tool" (Liu PR 2007) [3].

The cross-language image retrieval campaign ImageCLEF (Clough AMIR 2006) [4] (Clough EMMIR 2007) [5] aims to evaluate different approaches of text and content based retrieval methods. The focus is set on a (natural) language independent solution for image retrieval, which exploits both textual annotations as well as visual features. This effort also shows quite clearly the need for a powerful image retrieval system.

This paper introduces one approach to solve some of the claims stated above. It describes a query language which is designed to be reasonably user friendly and allows the integration of high-level semantics and low-level feature extraction in a single query.

Taking a look at current full text retrieval engines, it reveals the main differences to CBIR engines. Image retrieval

*Address correspondence to this author at the Department of Informatics, School of Computing and Engineering, University of Huddersfield, Queensgate, Huddersfield HD1 3DH, UK; E-mail: r.p.pein@hud.ac.uk inevitably contains fuzzy aspects. A search based on image features usually produces a list of results with decreasing similarity. In contrast, a full text search can determine separate hit and miss lists, even if some fuzziness is added by language analysis (e.g. ignoring word endings).

Such a language must tackle the tasks of synthesizing simple result sets with fuzzy sets (Fagin PODS 1996) [6] as well as keeping the final result in a maintainable size. The latter requirement is important because every similarity above 0.0 is somehow part of the hits.

At the same time, query composing in CBIR environments is often much more difficult as there are no keywords for low-level features. The query language presented in this paper is rooted in the established area of text retrieval and is extended by essential CBIR related additions.

\section{RELATED WORK}

This section surveys some related research that are dealing with query composing in information retrieval and using different ways of describing content.

\section{Query Language}

The Lucene Query Language (Apache Lucene ASF 2006) [7] is a full text retrieval language. The Lucene library includes a parser which converts a query string into a query object. This object represents all query details and the search engine generates the result based on it. This language is not suitable to handle fuzzy results out of the box, but provides a simple and clear structure. It allows boolean and nested queries as well as the definition of document fields. These fields hold some meta information (i.e. title, content, author, etc ...) and can be used to compose reasonably complex queries.

With the development of object-oriented DBMS the ODMG-93 (Cattell SIGMOD 1994) [8] standard emerged. The OQL query language (Alashqur VLDB 1989) [9] has been created. It combines SQL syntax with the OMG object model. An interesting extension to this language is called 
FOQL (Nepal ICDE 1999) [10]. This language extension tries to capture fuzzy aspects which are required for CBIR applications. The FOQL approach is to attach a set of matching-methods to each stored objects. These methods are used to match any two objects of the same kind in a specific way. The resulting similarity is somewhere between 0.0 (no similarity) and 1.0 (identity). The newly introduced data type is called Fuzzy-Boolean. In addition, the result can be limited by a threshold defining the minimum similarity.

Another query language is OQUEL (Town CBAIVL 2001) [11] (Town IVC 2004) [12] which is designed to be user friendly. It is based on a simplified natural language and an extensible ontology. The system extracts a syntax tree from the query to retrieve images.

\section{Data Description}

The feature vector paradigm states a plain list of several float values to create a vector. But looking at any random technique it reveals that features may be composed in many different ways, e.g. containing probably complex data structures. These structures need to be mapped to the query language.

The language MPEG-7 (Martinez IEEEMM 2002) [13] is rather a multimedia description than a query language. It is an emerging standard used in multimedia archives, often containing high level semantic information. Using an XML based language for typed queries appears to be very unhandy and overly complex.

A possible alternative is the minimalistic approach in JSON. This sub set of JavaScript is an important part of the current Ajax technology. JSON is intended to be a simple data interchange format with minimal overhead.

\section{METHODOLOGY}

The proposed query language is based on the Lucene Query Parser (Apache Lucene ASF 2006) [7] which defines a common language for full text search. It is intentionally chosen to provide beginners with a simple and familiar syntax. The language allows queries similar to those used in traditional search engines and the parser is generated by JavaCC.

This approach tries to merge the design principles of different languages. Some are like OQUEL (Town CBAIVL 2001) [11] where queries are kept as simple and natural as possible. Others like SQL define a strict grammar to be highly machine readable.

There are two changes made to the Lucene grammar to fit the requirements of an extensible feature vector based query language: fuzzy related operators and a nested twolayer grammar.

The previous boost parameter for terms has been extended to multiple TermParams allowing additional control of fuzzy result sets. To provide a high extensibility the grammar is split into two different layers.

The basic layer is parsed and interpreted by the search engine directly. Here the grammar is predefined and fixed. Users may specify which meta information should be searched for by using fields. Images hold other fields than normal text documents, typically EXIF and IPTC information. In the near future, this information may be replaced by the XML based XMP (Riecks IPTC 2005) [14]. Additionally, a CBIR environment provides one or multiple feature vectors holding low-level information about the pixels. These feature vectors can be added by plug-ins, each one having a unique identifier which is the field name for content based queries. The difficulty now lies in specifying how the query feature vector is entered. There are at least three different ways possible:

- ID of an image stored in the repository

- URI of a query image

- specification of the feature vector itself

The simplest way is to use an existing image for a query (query-by-example). Images already in the repository have the prepared feature vector available. Specifying the URI of an image requires the engine to load the image and to extract the feature vector. The most advanced and complicated way is to let the user specify a feature vector in detail.

As a custom feature vector may contain any kind of proprietary data, offering an all-embracing language is not possible. Thus a second layer is added to the query language. A Term may contain the string <FEATURE START $>$ [ $<$ FEATURE CONTENT $>$ ] $<$ FEATURE END $>$. The parenthesized part <FEATURE CONTENT> is extracted by the search engine and passed to the responsible plug-in. The plug-in is fully responsible for parsing and interpreting this string to return the object representation of the feature vector.

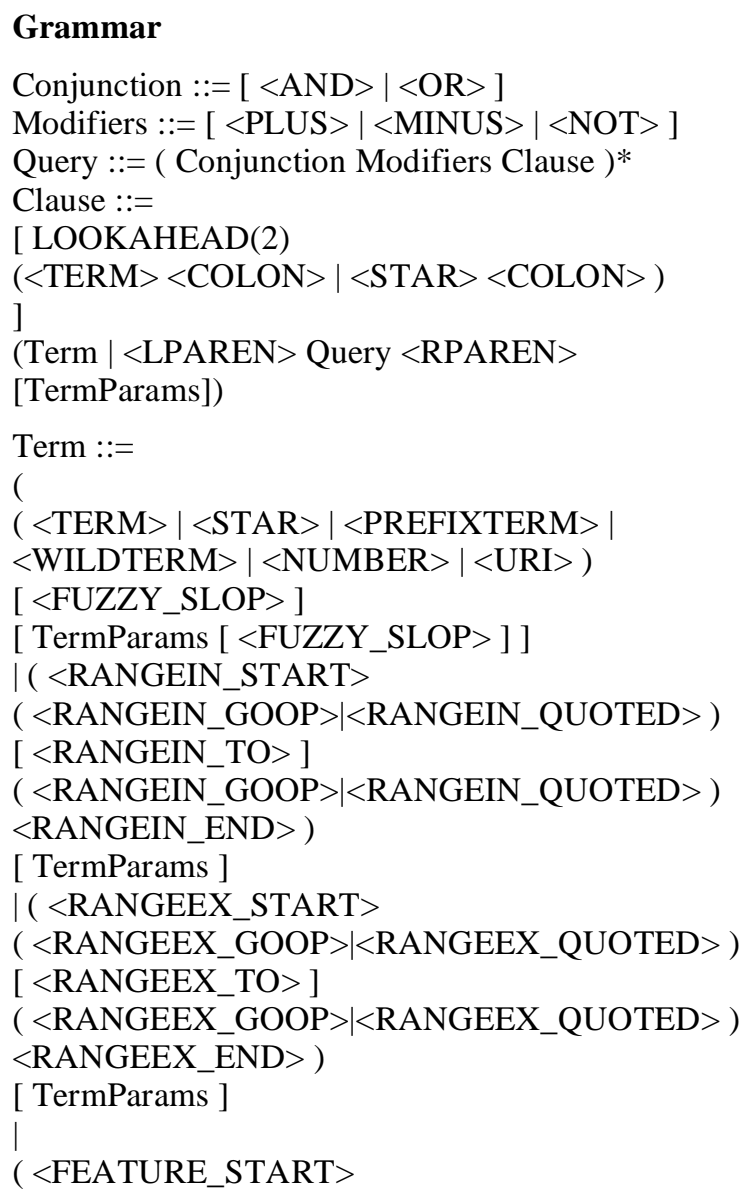




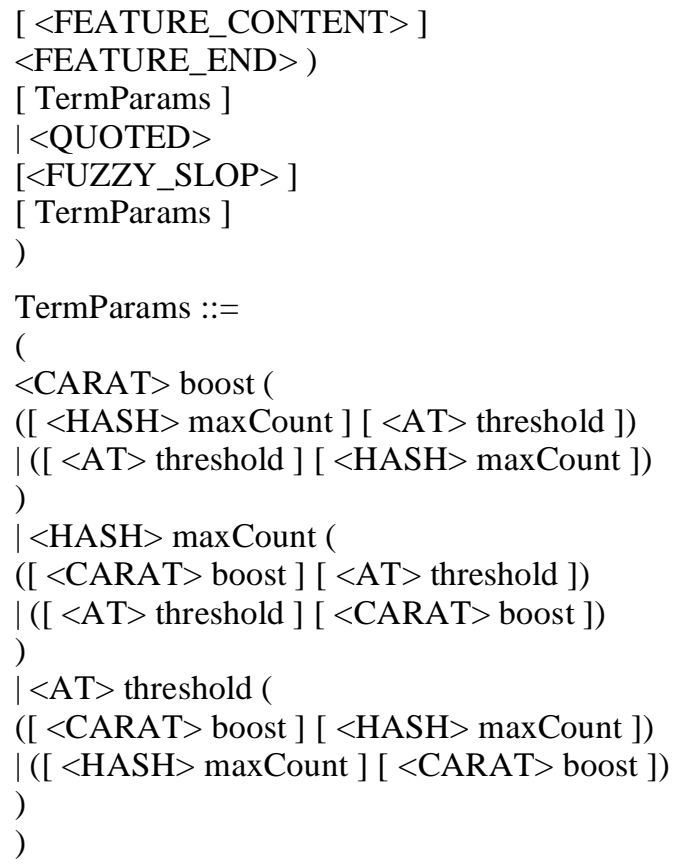

\section{Operators}

The main difficulty of combining sub results from a CBIR system is the fuzzy nature of the results. Some simple features with filtering character (e.g. keywords) deliver a rather clean set of hits. But it is essential to have a a fuzzy model for merging these with highly similarity based features. Those results are usually a sorted list (Fagin PODS 1996) [6] (Ramakrishna ADC 2002) [15].

The approach by Fagin (Fagin PODS 1996) [6] interprets results as graded sets, which are lists sorted by similarity and set characteristics. He uses the basic rules defined by Zadeh (Zadeh WSPC 1996) [16]:

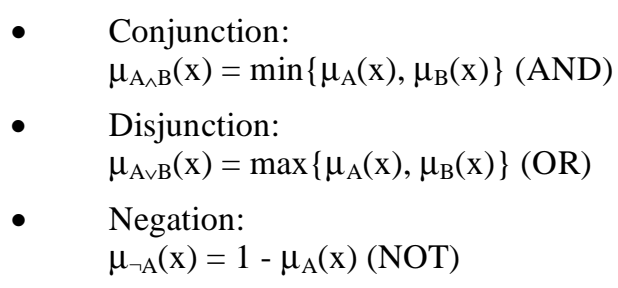

The text retrieval concept of boosting single terms by any float value is adapted to the extended engine. Before merging sub results, the similarities are boosted as specified to shift the importance into the desired direction.

An additional acknowledgement to the fuzzy nature is the use of additional set operators to keep the results at a reasonable size. The minimum similarity is a value between 0.0 and 1.0 and forces the engine to drop all results below this similarity threshold. As the efficiency of the threshold highly depends on the available images and features, a maximum size parameter limits the result to the specified size.

\section{Plug-Ins}

The plug-in concept of the retrieval framework described in (Pein ICCS 2007) [17] allows the definition of any new feature. To make such a plug-in available in this language, only a few requirements need to be met.
The plug-in needs an identifier which is automatically used as a term field. With this information it is already possible to formulate queries containing an example image (either by internal id or URI).

The tricky part is to develop syntax for user defined feature vector information embedded in a query. As features can be arbitrarily complex, it is intended to support a simple default language like JSON. Otherwise the embedded data string of a query is forwarded directly to the feature plug-in where it needs to be converted into a valid feature object.

\section{Wildcards and Ranges}

Wildcards and ranges can be used to express uncertainty or to allow the search engine to be less strict during retrieval. The meaning of those concepts depends on the described feature. Some features may well benefit, but for others they may not be required.

In text retrieval, wildcards stand for letters in a word that don't have to match an explicit query. In the example case of a RGB mean value, a wildcard can express, that a certain colour channel does not need to be considered. For spatial features it can be useful to define regions of interest as well as regions of non-interest.

Ranges are an intermediate concept between concrete queries and wildcards. They are used to specify a certain space where parameters can be matched. Searching for images with a creation time stamp is only feasible, if a range can be specified. It is very unlikely that the searcher knows the exact time, especially when it is extremely accurate (i.e. milliseconds). In such a case, usually a time span is provided (e.g. "between 03/06/08 and 10/06/08" or "within the last week"). Analogous, image features such as the trivial RGB mean could specify a tolerance range for each colour channel.

Unfortunately, these definitely useful concepts cannot be fully generalized. At this point, the plug-in developer needs to decide how to address them. Taking the RGB means of an image, the user could specify an array like "[36, 255, *]". In this case the results should contain some red and dominant green. The rate of blue does not matter at all. Putting some more effort into the feature abstraction, a more convenient query like "some red and very much green"is also possible. This lies in the responsibility of the plug-in developer.

\section{Examples}

The following examples demonstrate the use of different language constructs, where the "keywords" field is the only text based one.

1. IPTC keyword: keywords:oystercatcher

2. external image, similarity at least $95 \%$ : histogram:"file://query.jpg”@0.95

3. wavelet of three images by internal ID: wavelet:(3960 39413948$)$

4. two histograms, maximum of 10 results each and the first one boosted by 2.0: histogram:3963\#10^2.0 OR histogram:3960\#10 
5. spatial histogram without the 50 most similar images to image 190: spatial histo:5456 -histogram:190\#50

6. mean colour with embedded feature and filtering keyword: rgb mean: $(\$[200,50, *] \$)+$ keywords:car

Example query 1 is a simple text based query based on the IPTC meta information. It works exactly as every common full text retrieval. The field 'keywords' is derived directly from the IPTC data and other fields such as 'title', 'author' or 'createdate' are also available.

More interesting in this context are queries allowing CBIR relevant features. The fields are picked by the feature identifier and processed in the plug-ins.

Number 2 searches for similarities based on a histogram plug-in that implements a feature proposed by Al-Omari and Al-Jarrah (Al-Omari DKE 2005) [18]. An URI to an image is specified which is used for query-by-example. The engine loads the image and extracts the required query feature. The final result is limited to images with at least $95 \%$ similarity.

Query 3 calls the wavelet plug-in which is an implementation of a feature by Jacobs et al. (Jacobs ACS 1995) [19]. The query contains three internal image IDs. The engine performs three parallel sub retrievals and merges the three result lists by default with OR. Using the IDs shortens the query string itself and allows the engine to load the prepared feature vectors directly from the persistence. Because of the fuzziness in CBIR it is not clear how many results are returned when giving a similarity threshold. Dependent on the quality of the feature implementation and the repository size, many thousands of images could have a similarity above a given threshold. This is usually a waste of resources because users want the result to appear in the first few hits, say the first result page.

Query 4 presents the second way to keep the result size tight. Here the result set of each term is cut off after a maximum of 10 results. This restricts the maximum result size to $10+10=20$ images. Additionally the first term is boosted by factor 2 , giving it a higher weight than the second term.

Having access to multiple feature plug-ins opens an interesting new field to composing CBIR queries. Different features often mean very different result sets. The NOT modifier in query 5 shows an example how to remove unwanted content from the result. First the engine searches for the feature spatial histo, which is a histogram with additional information about spatial colour distribution (Pein IKE 2006) [20]. As this query might return several images which do not correspond to the wanted context, a NOT term filters out the 50 highest results similar to an unwanted result which are hopefully very similar in the simpler histogram space.

Finally the conjunction of the two different worlds is done by example 6 . The first term searches for the content based $\mathrm{rgb}$ mean. The embedded part within the brackets is interpreted by the simple rgb mean plug-in, where the three values stand for red, green and blue. The desired values for red and green are defined and the blue colour does not matter at all. Because this low-level feature is far too simple for efficient retrieval, a second term is specified. In this example the keywords field is mandatory (AND) and has a filtering effect. Only images containing the keyword "car" are allowed to be in the result.

\section{System Design}

In order to test the new language in a meaningful context, it has been attached to the previously developed CBIR prototype (Pein MT 2008) [21]. This program was lacking a flexible interface to process complex queries. It was only capable of processing queries containing a set of weighted features. Concepts such as boolean joins were impossible to formulate. Plus, the interface required the use of some proprietary query objects. Adding a query language opened up a range of new possibilities.

Most retrieval systems follow a simple workflow cycle (Fig. 1) (Pein CIT 2008) [22]. A successful retrieval is performed as follows. Users submit a query to the engine to trigger a search and receive a set of results. Those results may be satisfying and the user finds the required information. If not, the user may navigate through the results and eventually refines the previous query to trigger a new search. In this paper, the stages from the query to the results are examined in more detail (Fig. 2).

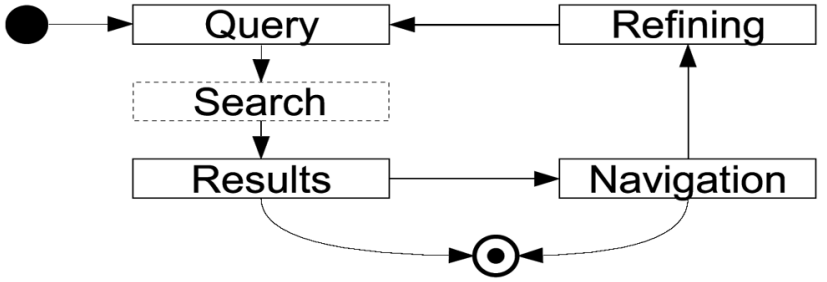

Fig. (1). Generic Retrieval Workflow (Pein CIT 2008) [22].

\section{Abstract View / Retrieval Workflow}

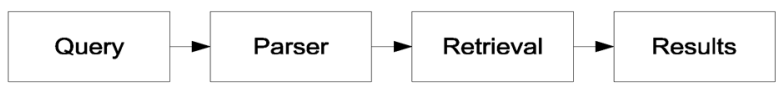

Framework Components

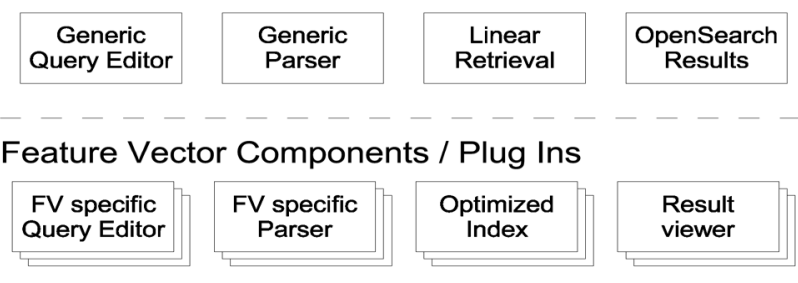

Fig. (2). Layers in the Retrieval Process.

At a very high abstraction level, a simple retrieval system receives a query from the user, parses it somehow to understand the meaning, gathers the most relevant documents and finally returns them. This workflow is very common and can be offered by a generic framework, which simply offers all the basic functionality required. Those framework components do not have to be specialized. They only need to understand basic input and generate basic output. All the details and optimizing are meant to be implemented in exchangeable plug-ins. 


\section{IMPLEMENTATION}

The design proposed above has been implemented in Java. The prototype uses a modified version of the original Lucene Query Parser. The parser analyses an input string and converts it into the corresponding Query object. Dependent on the terms given, the Query is composed of different clauses, such as an array of boolean clauses or may be even nested.

For the new parser, some amendments are required. A block for additional term parameters and the encapsulation of arbitrary strings have been added. In the current version, the parser ignores everything between the opening brackets " (\$" and the corresponding closing ones " $\$)$ ". This part of the query is stored in a special sub query object as a simple string. At a later stage, this string gets parsed by the corresponding module to create an object instance, that can be used for the partial retrieval itself.

\section{Query Objects}

The retrieval software works internally with well defined query objects rather than a query string. This ensures that the query can be easily processed and does not contain syntactic errors.

In the Lucene library, queries are composed of different object types. The basic class is the abstract Query. It contains a boost parameter and either a simple Term (TermQuery) or a nested Query. A commonly used implementation of the nested query is the BooleanQuery, which contains a list of clauses. Each Clause instance wraps a query and an occurrence parameter (MUST, SHOULD, MUST NOT).

The proposed query language requires a set of additional classes to express the special needs of CBIR. Opposing to normal terms, the new classes are able to store information such as URLs, IDs or a FeatureVector instance. The latter needs to create the feature information from the embedded string. To achieve this, it calls the parsing method of the corresponding plug-in.

\section{Parse Trees}

Based on the grammar, the parser generates a hierarchy of sub queries wrapped up in a single root query object. By traversing the tree, the sub results can be merged accordingly. This section shows the decomposition on a relatively complicated query. The images used in this example are part of the Caltech-101 collection (Fergus CVPR 2003) [23].

(

(

histogram:"file://query.jpg" OR

rgb_mean: $(\$[200,50, *] \$)^{\wedge} 2.0$

)@0.8

-wavelet:(89244345)@0.9

+keywords:airplane

)\#100

Verbally, this query can be read as follows:

"Find me images, that have a similar histogram as the sample image query.jpg OR have a mean colour close to 200 red and 50 green. The blue channel can be anything. Rank the mean colour twice as high as normal. Both sub results should have at least a similarity value of 0.8 . Please remove any result, which has a minimum wavelet similarity of 0.9 to the images 89, 244 and 345. Every result must be annotated with the keyword airplane. Give me not more than 100 results in total."

After parsing, the query string is converted into a parse tree that contains all of the relevant concepts (Fig. 3). The root node is represented by a Query, which is the single data object that is processed by the retrieval core. Each leaf is a Term, representing a partial search, which generates a sub

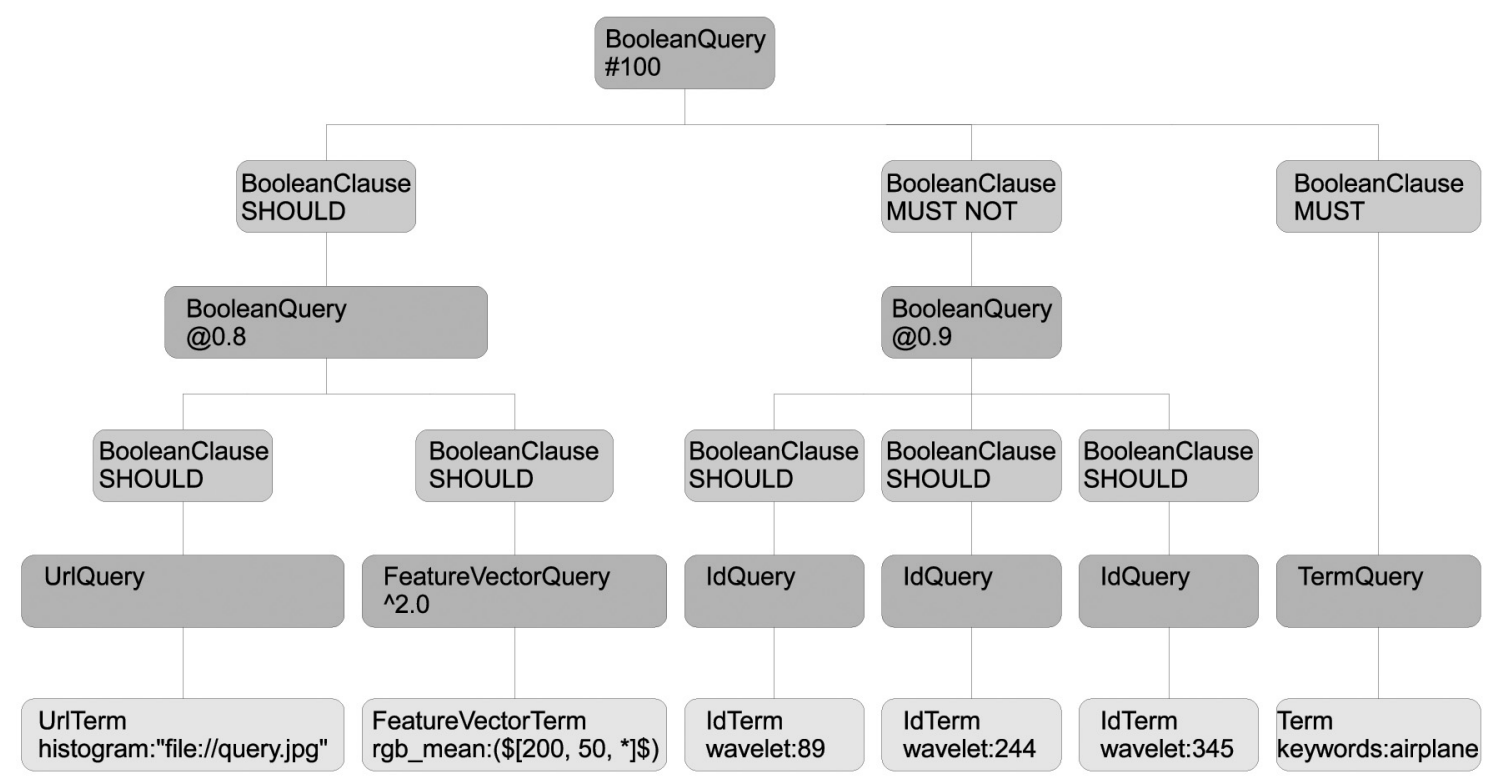

( ( histogram:"file://query.jpg" OR rgb_mean:(\$[200, 50, *]\$)^2.0 )@0.8 -wavelet:(89 244 345)@0.9 +keywords:airplane)\#100

Fig. (3). Parse Tree of a complex Query. 
result. The tree structure in between represents the rules how to merge the sub results into a final one.

The search engine then traverses the tree and generates the answer to this particular request. At this point, it is advisable to integrate a query optimizer to reduce the response time. In the current prototype, some straightforward query optimizing already takes place.

First, the MUST clauses are processed, then the SHOULD and finally the MUST NOT clauses. This allows for an early reduction of the search space, which is especially of importance, if no or only a slow index is available for certain features. Depending on the availability of indexes, certain other term could also be pre-drawn. The optimization strategy should always be aimed at an early reduction of search space as well as preferring the use of fast indexes. The strategy used in this cases uses a strict definition of MUST and MUST NOT. If an image is not part of all the MUST clauses or part of a MUST NOT clause, it is removed from the final result. This approach is considered to be a useful trade-off between a perfect fuzzy algebra and speed optimizations.

In this case, the first term to be processed is "keywords:airplane". This triggers a keyword search, which is backed by a fast and efficient index, resulting in a list of matching images. As the parent BooleanClause is flagged as MUST, the final results of the query can only be amongst those sub results. Assuming, that only about $1 \%$ of the repository is related to the keyword "airplane", every subsequent linear search time can also be reduced to only $1 \%$ of the otherwise total scan time.

The second branch to be processed is the SHOULD clause on the left, that is split into a nested boolean query. One leaf contains a UrlTerm, pointing at an external query image and requesting a comparison based on its histogram. To process this part, the engine reads the image from the URL and extracts the histogram automatically. This search only needs to compare the query histogram with the stored histograms from the previous sub result.

The other leaf contains a FeatureVectorTerm. The string embedded between the " $(\$ "$ " $\$)$ " brackets is parsed by the rgb mean plug-in. In this case, the string stands for the three mean colour values red (200), green (50) and blue ("don't care" wildcard) of an image. Again, the search space is drastically reduced by the first sub result.

After both terms have been processed, the sub results are merged into a single one. Their combined similarity must be at least 0.8 , otherwise the image is removed from the result set. There is no "best" rule to merge the sub results. In the current prototype, the combined similarity is calculated by determining the weighted and normalized sum of the sub similarities. In this case, the rgb mean branch has a weight of 2.0 and thus gains a higher importance in the merged result.

The last main branch is flagged as MUST NOT and requires a minimum combined similarity of 0.9 . All of the three clauses contain a plain IdQuery with an IdTerm. They require a retrieval on the wavelet feature and use sample images from the repository by stating the image id directly. Again, the search space is already limited, not only by the MUST branch, but also by the SHOULD branch. It is only necessary to check the images contained in the previously retrieved sub result. The sub results of the middle branch are merged accordingly and cropped at a minimum similarity of 0.9 .

To generate the final answer, the MUST NOT results are removed from the temporary sub result. The last step required is to cut the sorted list after the 100 best hits.

\section{Alternative Data Representation}

The string representation of a query can be manipulated directly by users. This query string can be edited in every basic text editor without the need for any extended user interface. It also allows experienced users to access every aspect of the search engine directly.

As the query language is based on the Lucene tool kit, it always has an object representation of the whole query. This query object could also be created by a suitable front end. Such a tool eliminates parsing errors, because the query structure would always be bound to the components.

\section{XML}

The parse tree containing a query can be directly mapped to an XML hierarchy. Plug-ins could also specify their feature conversion into XML and back to. This allows for consistent XML files and simplifies the use of the MPEG-7. Below, the XML structure matching the example parse tee is shown:

<boolean-query max-count="100">

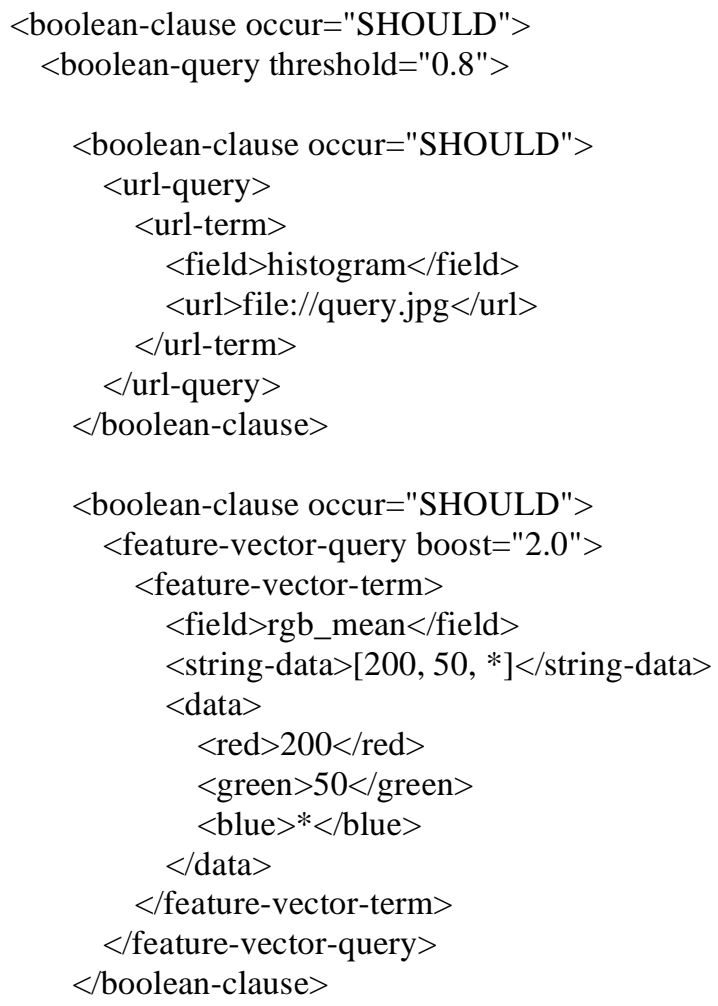

$</$ boolean-query $>$

$</$ boolean-clause $>$

<boolean-clause occur="MUST_NOT"> 


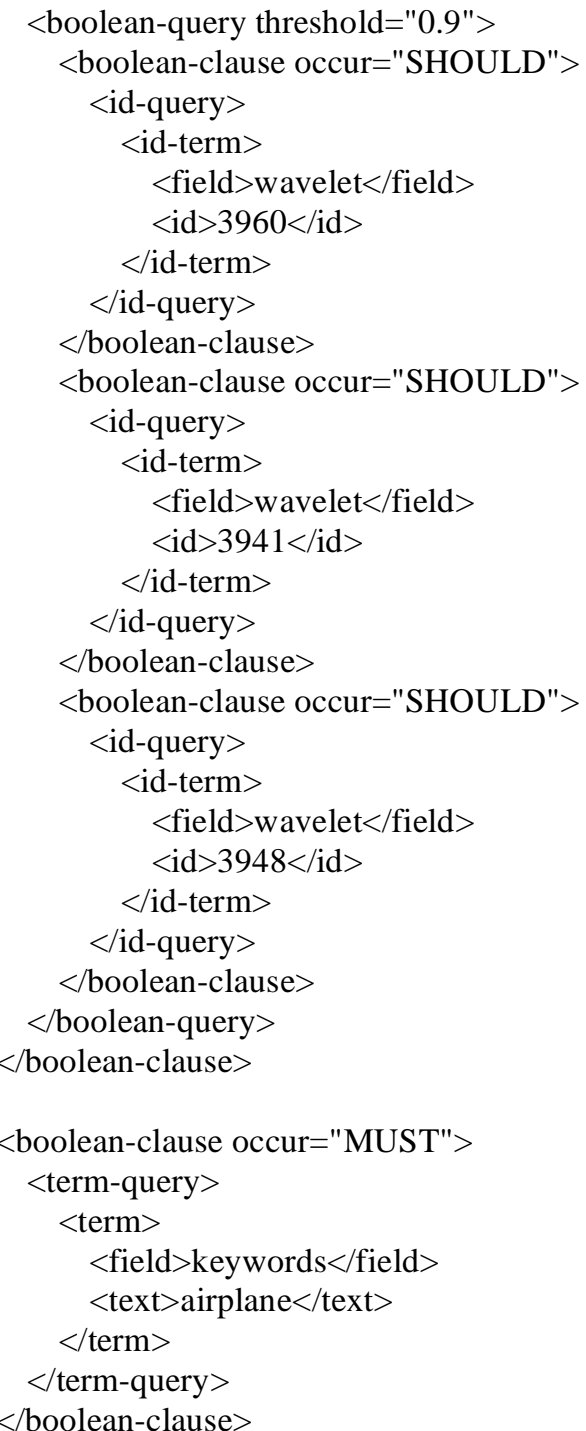

$</$ boolean-query $>$

This XML data contains the same information as the example query string. Clearly, this format is much more verbose than the suggested query language. Being probably less readable for humans, its advantage is the standardized format. The XML code does ot require a special parser to be processed or validated by any program.

One example of generic and specialized data representation is contained in the XML query above. The featurevector-term for the rgb mean plug-in shows two alternatives. In the generic case, the string-data is left untouched. This is the output generated by the main parser. To extract the real meaning of the data string, it needs to be processed by the corresponding plug-in. The resulting data tag would then contain each piece of feature data separately.

\footnotetext{
$<$ feature-vector-term $>$

$<$ field $>$ rgb_mean $</$ field $>$

$<$ string-data>

$[200,50, *]$

$</$ string-data $>$

$<$ data $>$
}

\author{
$<$ red $>200</$ red $>$ \\ $<$ green $>50</$ green $>$ \\ $<$ blue $>*<$ blue $>$ \\ $</$ data $>$ \\ $</$ feature-vector-term $>$
}

\section{Visual Query}

A clearly structured query language like the proposed one can optionally be mapped to a visual representation to guide the user. The resulting graphical user interface helps to assemble queries that are syntactically correct, displays query images, provides a canvas for query-by-example and may also support to adjust feature plug-in specific parameters.

Fig. (4) shows the visual query composer of the prototype, where the example query has been assembled from multiple clauses. Every clause of the parse tree is modelled by a window. Each clause window contains several options to choose the occurrence, the added parameters, a field name and the query type. Textual queries usually contain a generic term with one ore multiple keywords.

Queries for CBIR can manage a query URL, a specified feature, the id to an existing image or a canvas to draw a query image. Clause windows specifying a query image can directly display a small preview image to provide feedback what is going to be searched. Windows containing a feature description can either show the data fields directly (e.g. red, green, blue) or a convenient editor (e.g. a colour chooser).

\section{TESTING}

\section{Language Features}

There are only a few query languages which try to tackle the task of merging aspects of full text and CBIR retrieval. These languages need to address the requirements of fuzzy decisions between hit and miss. Above, several important features are discussed. Below, some languages are checked against them. Additionally, the proposed query language is tested against some synthetic retrieval tasks to evaluate its expressiveness in a reasonably realistic environment.

\section{Test Repository}

The testing repository consists of 6480 images from three different sources. Each source contains several photographs with certain topics. The level of annotation for each source is varying. As the images were from German sources, most image annotation is in German.

The first set of 415 images contains images from the cities of Dublin and Liverpool. There are many buildings and bird views as well as scenes from a football stadium, without any annotation. The only textual hints can be taken from the file names and paths.

The second set contains 5399 images and is by far the largest part of the repository. Apart from 417 photographs from a botanical garden and some large animals, the main content is birds. Most images contain a bird in the centre part and the background is dominated by water, grass or sky. Almost every photograph contains IPTC annotation with the name of the depicted animal. Some keywords also denote the location where the photograph was taken. 


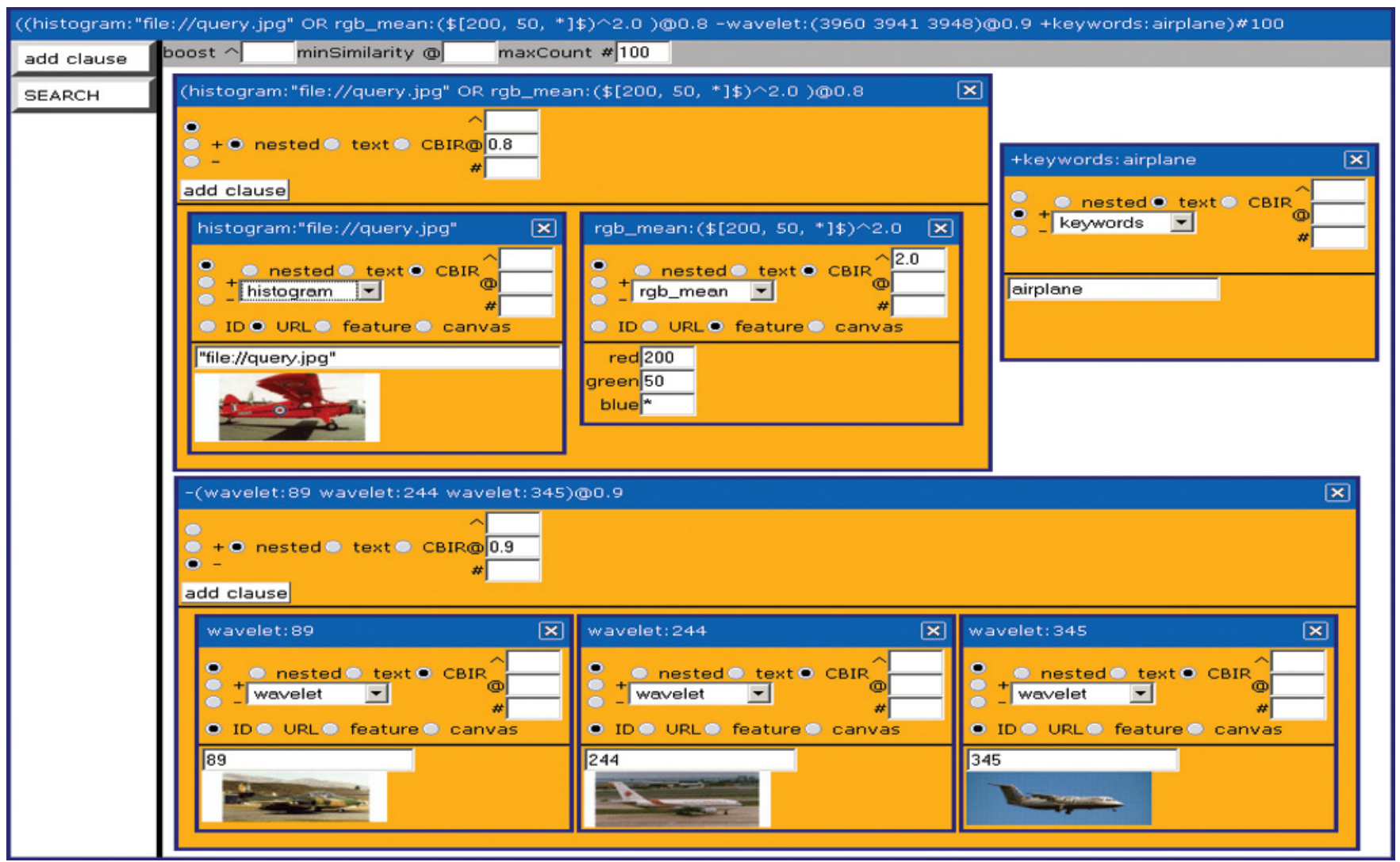

Fig. (4). Visual query composer.

In the third set there are 666 images from 4 different locations, Beijing, Shanghai, Dubai and the USA. The images have an average of 3 keywords describing the location and the content. Similar to the first set, most pictures show buildings or landscapes.

\section{Single Feature Test Cases}

To assess the retrieval quality of single features, two image series from the repository are chosen. In these series there are several images with similar content as well as some more difficult changes. Each feature plug-in is tested by taking some of the images and afterwards precision and recall are determined on the results.

The first testing set contains 14 closeup views from Highland cattle (Fig. 5). The images are dominated by the typical auburn fur and some water in the background. In human perception there are no other images with similar content in the repository.

The second testing set containing 57 images of a meadow pipit (Fig. 6) is much more challenging. More than 50 percent of the repository images show birds. This image set consists of 7 different sceneries. The feature vectors should at least be able to find the images of the same series. As none of the implemented feature vector plug-ins is capable of identifying a small bird in the central part of images, it is expected that all of them will get to serious issues to find images depicting the same bird from another series.
For testing some of the images from the image sets are taken as query. For the first set only one image (Fig. (5a)) is used, because of the relative high similarity within the set. From the second set always the first image of each series is chosen as query except from the single one (Fig. (6b)) are used for the query. Sometimes the first image is obviously not the best choice but these test cases try to capture real and maybe unclear conditions.

\section{Multi Feature Language Test Cases}

In this testing series it is attempted to create queries which are more efficient than the simple queries from the section above. To allow a "natural" progress of query composing and refining, the IDs of the other related images are treated as unknown. Each related ID needs to be present in a previous result set in order to be used in the next query. Each retrieval starts with the query image.

A search by keywords would result in the correct small subset of 14/57 images. Using CBIR on the remaining images would be pointless for testing. The impact of keywords is examined in the user survey below.

The basic search strategy is as follows: First the query image is used with every basic single feature. Only the first 50 hits are browsed for found relevant images. Starting with the best performing feature, the IDs of all relevant images are used in new queries. Other images found are also used 


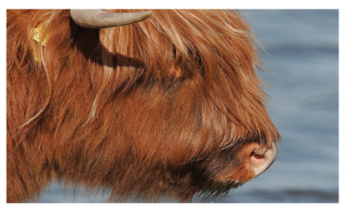

(a)

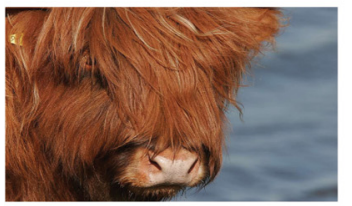

(f)

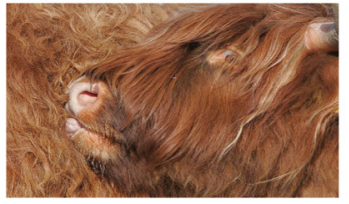

(k)

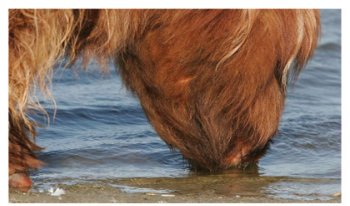

(b)

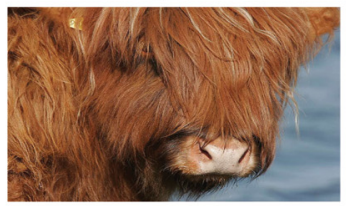

(g)

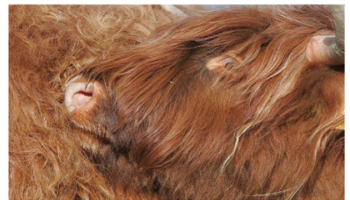

(I)

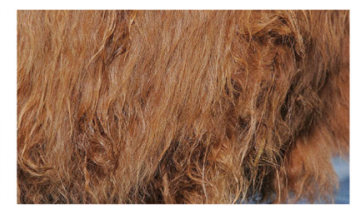

(c)

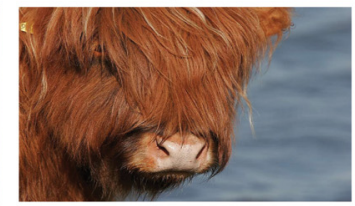

(h)

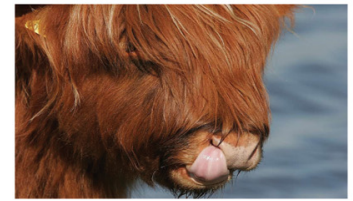

(m)

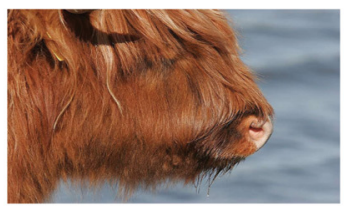

(d)

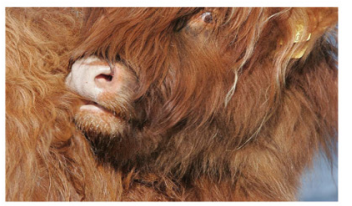

(i)

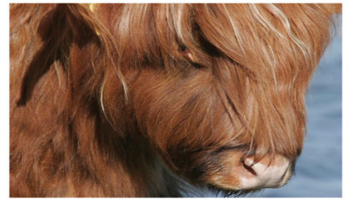

(n)

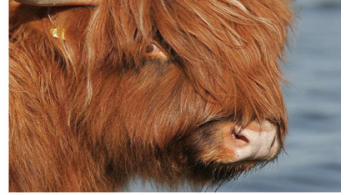

(e)

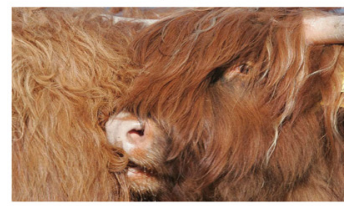

(j)

Fig. (5). Selected meadow pipit images.

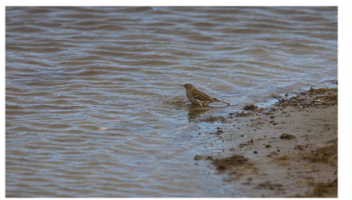

(a) 6 images

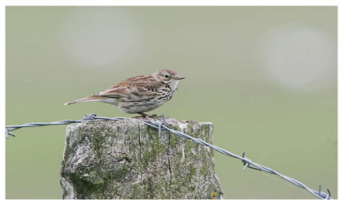

(f) 9 images

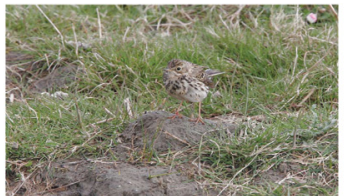

(b) 1 image

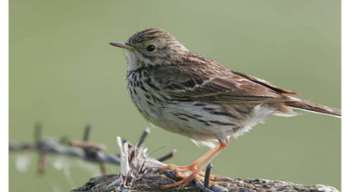

(g) 3 images

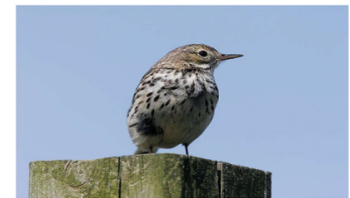

(c) 13 images

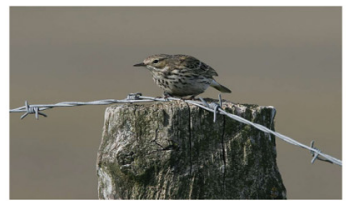

(d) 17 images

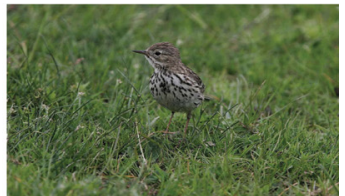

(e) 8 images

Fig. (6). Cattle images.

for querying. If still some images are missing, the same procedure is repeated with the other available features. The found images are then combined in a single query and optimized for a high recall. In difficult cases the results are browsed in depth and the approximate ranking of the related images is determined.

If other image series with a high similarity clutter the results, it is attempted to filter them out with a NOT term. The final query containing all targeted images is then cut to the minimum size possible.

\section{User Survey}

A first small-scale user survey has been carried out to evaluate the language in the context of the related master thesis (Pein MT 2008) [21]. For this survey, the same repository as described above is used. It has been carried out with 5 testers with at least basic experience in computing sciences. The testing prototype only offered a plain HTML page with a single input line for textual queries. The visual query composer was not available at that time.

Each tester got a short introduction into the query language and some technical basics of the different available features. During the procedure the testers were allowed to ask for further advice concerning the system abilities and usage. It is important that the direct influence of the test supervisor is kept as low as possible. He must not give direct hints how to solve a task. The only support allowed is to mention basic technical possibilities available or workarounds to avoid bugs.

\section{Tasks}

The tasks demand both CBIR and keyword based approaches. The basic tasks were: retrieving images based on a textual description or visual examples, tracking a given example image, optimizing queries (high Precision/Recall) for a specific content and ascertain the name of birds from given images.

The first task is starting simple to let the testers get used to the search engine. First some blue images need to be found. This could be easily done by using the RGB Mean plug-in and the word "blue" or alternatively the

RGB value $[0,0,255]$. The second subtask is basically the same with "white" or [255,255,255]. The two remaining subtasks are not trivial, because no query-by-sketch module is available. The testers need to find examples directly from the repository and then use them for querying. It is expected 
Table 1. Languages Compared

\begin{tabular}{|c|c|c|c|c|c|c|c|c|c|c|}
\hline 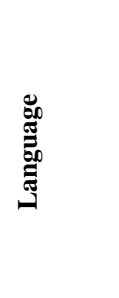 & 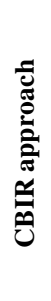 & 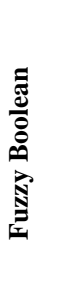 & 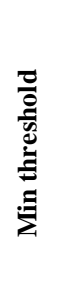 & 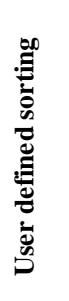 & 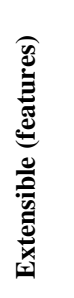 & 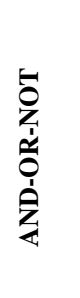 & 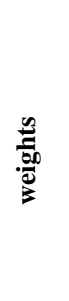 & 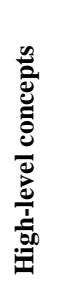 & 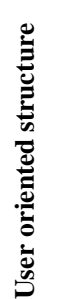 & 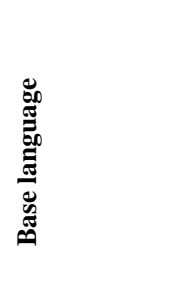 \\
\hline This & & $\mathrm{Y}$ & $\mathrm{Y}$ & $\mathrm{N}$ & $\mathrm{Y}$ & $\mathrm{Y}$ & $\mathrm{Y}$ & $\mathrm{N}$ & $\mathrm{Y}$ & Lucene \\
\hline FOQL & & $\mathrm{Y}$ & $\mathrm{Y}$ & $\mathrm{Y}$ & $\mathrm{Y}$ & $\mathrm{Y}$ & $\mathrm{Y}$ & $\mathrm{Y}$ & $\mathrm{N}$ & ODMG/OQL \\
\hline OQUEL & & $\mathrm{Y}$ & $\mathrm{Y}$ & $\mathrm{N}$ & $\mathrm{N}$ & Y & $\mathrm{Y}$ & $\mathrm{Y}$ & $\mathrm{Y}$ & None/natural \\
\hline
\end{tabular}

that a random search produces a suitable query image and that the Spatial Histogram feature or promising keywords are used.

The second task is to spot predefined images. All of them have some keyword annotation. One image (a Chinese stop sign) is very easy to find. The keywords "stop", "sign" and "china" all narrow down the search space drastically. Additionally a search for red content can be helpful. The other images require to use less obvious keywords and maybe CBIR. In one case, some background knowledge in ornithology was beneficial. Testers knowing that a "great crested grebe" was depicted, could find this image by keyword. Others need to browse a bit more and do some CBIR.

Task 3 is similar to the previous one. The difference is that instead of a specific image, several similar images need to be found. The first image was one of 441 "oystercatcher" images in the repository. Knowing the name is already very helpful, but from the remaining images the ones with the highest similarity need to be found. A combination of keywords and one of the CBIR features returns a nicely sorted list. The second image was much harder to find. It shows a brown cathedral, that has been photographed from several different angles and distances. The keyword "liverpool" reduces the search space to 315 images. This city name could be found by recognizing the building or by looking at the keywords of random results.

After having collected some experience with the system, the testers are challenged to do some query optimizing in the fourth task. The first subtask to find pictures of the Great
Wall of China was trivial when using the keywords. Alternatively a CBIR query could be composed based on random results. The second one requests images from a desert is more difficult, because the real keyword was "sandy-desert" (in German "Sandwüste" instead of the more general "Wüste") and requires the use of a wild card or the correctly spelled keyword. Alternatively, the retrieval by yellow content or less specific keywords like "Dubai" lead to success. The final sub task was to find city skylines. The search for the "city" only returns pictures from the "forbidden city" in Beijing. Here it is advisable to either use known city names from the repository or by picking random images.

The final task was a bit tricky. The testers are supplied with a small image of a certain bird. Either they know the name or they need to do some retrieval work. There are several ways to find the images. This required some kind of freestyle retrieval with no best solution or short cut (except from knowing the bird names).

\section{RESULTS}

\section{Language Features}

Table 1 compares some query languages and lists which important requirements are met. Each language addresses all of the fuzzy aspects. They only differ in "comfort functions". The language proposed here lacks a user-defined sorting and the direct implementation of high-level concepts. FOQL is very expressive, but is very verbose in comparison. OQUEL is a very high-level language and its abilities ultimately depend on the ontology used.

Table 2. Single Features Recall, Recall for the First 50 Hits

\begin{tabular}{|c|c|c|c|c|c|c|c|}
\hline & $5(a)$ & $6(a)$ & $6(c)$ & $6(d)$ & $6(e)$ & $6(f)$ & $6(\mathrm{~g})$ \\
\hline & & & & & & $\varepsilon^{2}$ & this \\
\hline RGB Mean & 0.85 & 0.0 & 0.83 & 0.13 & 1.0 & 0.0 & 0.0 \\
\hline Histogram & 0.23 & 0.8 & 1.0 & 0.19 & 0.29 & 0.0 & 0.0 \\
\hline
\end{tabular}


Table 3. Single Features Similarity, Similarity for the $50^{\text {th }}$ Rank

\begin{tabular}{|c|c|c|c|c|c|c|c|}
\hline & $5(a)$ & 6(a) & $6(c)$ & $6(d)$ & $6(e)$ & $6(f)$ & $6(\mathrm{~g})$ \\
\hline RGB Mean & 0.9939 & 0.9997 & 0.9992 & 0.9997 & 0.9975 & 0.9992 & 0.9992 \\
\hline Histogram & 0.9936 & 0.9837 & 0.9862 & 0.9972 & 0.9947 & 0.9971 & 0.9926 \\
\hline Spatial Histogram & 0.9943 & 0.9877 & 0.9880 & 0.9951 & 0.9969 & 0.9834 & 0.9937 \\
\hline
\end{tabular}

\section{Single Feature Test Cases} 3.

The results of the testing is summarized in Tables 2 and

Table 2 shows the recall values for a result set of $50 \mathrm{im}-$ ages. This size is chosen because it is assumed that 50 results are reasonably displayable at the same time. Sometimes a couple of relevant images still show up in later positions, but these may be already ignored by an impatient user (in fact, the user survey revealed, that single hits are often overlooked when scrolling quickly through the results). The query image is always at the first position and is deducted from the recall. A recall of 1.0 means that all expected images could be successfully retrieved. The value 0.0 however indicates a complete failure.

Table 3 lists the similarity values of the 50th image in the result. This value indicates how satisfactorily a feature is capable to avoid a false positive. The more images gain a high similarity, the more difficult it is to do the final ranking in a right way. In the end, the similarity is simply an indicator for the manner how the results should be sorted. The quality itself is determined by the ranking.

\section{Multi Feature Language Test Cases}

In this section a possible progress of query refining is listed. Each query image from the tests above is used as initial query to find all the other ones from the related series. It is aimed to gain the highest recall possible with the final query. During optimization, usually the hard limitation of images has been chosen (\#) to indicate the final result set size. In a repository that is assumed to be changing, it is recommended to use the more flexible similarity (@) restriction. Otherwise, hits may be pushed out by new false positives.

\section{Search for Image Series 5(a)}

The best results are achieved by using the simple RGB mean feature. This query already returns 12 of the 14 possible images among the 50 highest ranked results. Only two images are missing. To find them, the other images found are used for querying. With only two additional queries all 14 images are successfully retrieved, resulting in the optimized query:

(

fv_mean:4833\#40

fv_wavelet:4839\#5

fv_wavelet:4843\#5

)+42

\section{Search for Image Series 6(a)}

The second task is trivial, as the wavelet feature is almost perfect to solve it:

fv_wavelet:6424\#10

\section{Search for Image Series 6(c)}

Similar to the previous one this image series is easily found by a single feature:

fv_stochastic:6431\#37

Yet, some of the false positives can be removed by defining some NOT terms cutting away some of the unwanted content. Three NOT clauses already suffice to narrow down the result set from 37 to 21 images. Notably the last clause needs to be restricted to a total of 600 images. Otherwise this term would contain a relevant image and thus cut it away. The others default to a maximum of 1000 hits:

(

fv_stochastic:6431\#37

-fv_wavelet:4280\#1000

-fv_mean:4345\#1000

-fv_wavelet:5461\#600

) \#21

\section{Search for Image Series $6(d)$}

This one is the first challenging task. For this reason, all search iterations are described. The four available features all return some relevant images but they differ. In the first iteration, the user gets in 7 different hits in total:

fv_mean:6444 3 hits

fv_stochastic:6444 4 hits

fv_stoch_quad:6444 4 hits

fv_wavelet:6444 3 hits

Because the Wavelet feature does not add much new content the three other features are combined by SHOULD clause. The result is promising and contains 12 hits. Compared to the previous simple queries 6 new images are retrieved. In this series only 5 other images are missing:

fv_mean:6444

fv_stochastic: 6444

fv_stoch_quad:6444

Playing around a bit with NOT clauses reveals more images. This simple addition already caused a 15th image to appear: 
fv_mean:6444

fv_stochastic:6444

fv_stoch_quad:6444

-fv_wavelet:1476\#1000

Some exhaustive testing with lengthy queries finally revealed all 17 relevant images. Each NOT clause is first checked for positive hits. If it contains some irrelevant images and no relevant one, it is simply added to the query. The new query should now generate fewer results and still contain all previous relevant images. Else, the NOT clause is simply cut down to a smaller size:

fv_mean:6444 fv_stochastic:6444

fv_stoch_quad:6444

-fv_wavelet:1476-fv_wavelet:2753\#50

-fv_stochastic:2765\#200

-fv_wavelet:2003\#800

-fv_stochastic:5154\#400

-fv_mean:2588\#500

Based on the retrieved images each feature is tested with the other images for query. In this case a combination of three SHOULD-clauses proves to be highly efficient. Among the 17 hits only two false positives are contained. The optimized and reasonably compact query retrieves all 17 target images within 19 hits:

(

fv_wavelet:6460@0.75

fv_wavelet:6457@0.7

fv_stoch_quad:6444@0.995

)\#19

\section{Search for Image Series 6(e)}

Again this task is very easily solved by a single feature, but further optimization is possible. All 8 images are already among the first 50 results. Taking another image for querying shows that the 7 other images of the series are very closely related. The Wavelet feature easily retrieves all of the required images with a perfect precision. Only the initial query image cannot be found again without big effort. To get the perfect result set, the Wavelet feature is used to find the 7 closely related images and the single one is directly retrieved by id. If there would be more images similar, a feature should be used instead:

\section{(id:6461 fv_wavelet:6466@0.75)\#8}

\section{Search for Image Series $6(f)$}

Here the best result to start with is achieved by the Spatial Histogram. The first 3 relevant hits are in good ranks. The other images are too dark to be retrieved directly and the next relevant image appears at rank 390. Assuming that the user is persistent, he might have tracked the missing image and find its ID. Based on this ID the remaining images are very easily found by a single feature, in this case the Wavelet. Having this information at hand, the final query is short and concise. The precision is also very good and all 9 relevant images are contained in a result of only 10 hits.

(

fv_wavelet:6469@0.8

fv_wavelet:6472

)\#10

\section{Search for Image Series $6(\mathrm{~g})$}

The final retrieval task completely failed with the available features. As already visible in the previous section, none was able to find a single relevant image. An analysis of the result set showed that the best ranked image was by RGB Mean at position 800. The other features performed even worse.

\section{User Survey}

The survey was carried out with 5 volunteers aged between 20 and 40 years old. A questionnaire indicates that all of them were experienced computer users. Their expertise in digital photography and image processing was mostly slightly below average. A remarkable result is that all of them were very comfortable with Internet search engines, about average with local search engines and had almost no experience with CBIR engines. It was further revealed in the questionnaire, that no one has a very specific knowledge of any of the images stored in the repository. Only a single person declared that he knows more than average from China. These preconditions are quite useful, because it is not very likely that all of the testers succeed by only using the keyword search.

After a short training time, most testers were able to use both textual and content aspects in their queries. Mostly understandable features (colour mean, histogram) were used in combination with the query image IDs and the wavelet plug-in was often ignored. The simple rgb mean with its 3 values was a preferred feature. In some cases even the detailed histogram specification was tried out. As it was not allowed to draw query images, a popular approach was the use of random images to start with.

Most tasks were solved by the testers within less than 10 query iterations, but in some cases the available information and tools were not sufficient to ensure a quick success. The testers requested additional tools for query-by-sketch and complex feature composing.

\section{Tasks}

This section briefly describes the different approaches the testers chose. Except from single constellations, the testers succeeded in most tasks. Sometimes the search engine returned unexpected results. The testers learned the basic syntax very quickly.

The first two subtasks were solved by all testers in a single attempt. Interestingly some users chose the natural language and others chose to type in the appropriate RGB values. The remaining tasks were difficult without the ability to submit a query image. Some testers endeavoured to find suitable images by keywords, but failed because of missing annotation. Finally the random search combined with the Spatial Histogram was the most used approach.

The "stop" sign was nearly almost found immediately by a keyword search containing "stop" or "sign". One tester chose to use the RGB Mean in combination with the changing keywords "beijing", "china", and finally "sign". He succeeded after he set the keyword clause mandatory. Finding the second image, a lotus pond, turned out to be very challenging. The query with the RGB Mean to find green images 
returned hundreds of green images with the target image at rank 457. No one attempted to browse the results to this point. One person tried to filter out irrelevant images by keyword, but the annotation was simply too sparse. Only the hint that it might be a plant from china helped the testers to succeed very quickly. The "great crested grebe" was found very quickly. Either the testers knew the bird or they found it by a random search followed by a direct keyword search.

The "oyster catcher" series was found by either random search or the RGB Mean to find green images in combination with the folder name to narrow down the search space. Finding the cathedral from different angles took a long time. Using the RGB Mean to find the brownish building with the blue sky did not succeed, but sometimes it was helpful to find out the city's name faster than by random search. Having pinpointed a single image, it was used for relatively successful CBIR queries.

At task 4, everybody had an immediate success in finding the Great Wall of China by entering the correct keywords. The next subtask of finding images of a desert turned out to be more difficult. When entering the keywords a simple "desert" was not enough. One tester made use of wild cards and found all relevant images. Some other ones tried to find the images by RGB Mean and the parameter "yellow", which not immediately returns correct results. One user tried to alter the RGB parameters manually. After a few queries all of the users found the useful keyword "Dubai". To find city images, three users simply typed "city" and found the "forbidden city". Based on these images they either used different features with an adequate query image or they used the keyword "beijing". The other two testers exploited their previous results. One chose the keyword "dubai" and the other one picked some images for pure CBIR queries.

The last task turned out to be the most challenging one. Because no one knew any of the birds, there was no indication on how to begin. The favourite solution in all three cases was to start with a random search. Based on images with a promising content the testers massively used the CBIR features. With some luck the correct bird appeared in a result set and the name could be verified. Sometimes the result set reduction by NOT clauses was helpful. A captivating way to success was chosen by two users. Instead of giving up at this point, they used an external program to determine the mean colour values of the desired images and used them in a well directed RGB Mean query.

\section{Tester Comments}

The most wanted addition was tool support for query-bysketch or to upload example images. Also the keyword quality was often criticized. They were often far too specific or too general to be of real use.

In general the query language was accepted due to the prototype status of the system. However, the wish for a more comfortable user interface was obvious.

\section{Observations}

Throughout the tests it was observed that the searcher sometimes simply missed relevant images in the result set. In some cases the relevant image was already visible on the screen, but not noticed.
Concerning the available features, an aversion against the more complicated features is obvious. Actually only a single person used the Wavelet feature. Most testers preferred the RGB Mean for simple tasks and the Histogram for more complex ones. The offer with the natural language in the RGB Mean feature was generally accepted. In two cases, the testers opened an external program to determine the exact mean values of an example query image. Only two persons attempted to enter the 12 rather cryptic values for the Histogram.

The boost parameter was only used by a single person. The other parameters for result set restriction were not even tried out. This could be explained by the fact, that in most cases, the basic functionality was sufficient. Only the optimization task required its use, but the testers were hardly motivated to optimize their results further.

\section{DISCUSSION}

\section{Language Features}

The language presented in this paper represents the middleware of the previously described retrieval framework (Pein ICCS 2007) [17]. It is easily parseable and allows composition of any query that is supported by the framework. New feature plug-ins extend the language automatically by adding a new field. Currently the language does not inherently support high-level concepts. A plug-in for semantics could surely be implemented with some effort by collecting predefined queries with low-level features.

FOQL appears to be too complex and thus unsuitable for untrained users. Nevertheless many concepts like FuzzyBooleans and Fuzzy-Sets are valuable. It is possible to add any kind of feature by defining an appropriate method for object comparison. Due to its complexity and sorting ability the language is adequate in SQL like environments.

A closer view to OQUEL reveals some interesting features. The language itself has been designed to be easy to use. Users only need to specify the desired features in simple words (e.g. "people in centre")(Town IVC 2004) [12]. It is very close to a natural language, however the ambiguity of these requires additional attention and a well designed ontology. Concepts of this language help creating a convenient user interface.

\section{Single Feature Test Cases}

The results in Table 2 may seem confusing at the first glance. The performance of most queries is very poor.

One reason is that there are many images in the repository which are actually really similar to the queries but which are not contained in the related set for evaluation. If the query results would be judged by humans by asking which results are acceptable, they would often perform much better.

It is remarkable that the simplest feature of all, the RGB mean proves to be very efficient in some cases where more detailed ones completely failed. In return the similarity values always remain on a very high level (Table 3) leaving not much space to avoid false positives. 


\section{Queries}

\section{Query 5(a)}

Starting with the cattle, this image series contains several different images compared to the query image. The only advantage is, that the repository does not contain other images with semantically similar content. From an algorithmic point of view the changes of the background are challenging. Sometimes there is blue water visible and sometimes not. Interestingly the RGB Mean performs very well. The impact of the blue water is too small to cause trouble. Also the amount of this brownish shade of red seems to be not too prominent in the database.

The Spatial Histogram is very weak in this case. Only very few images of the relevant part are actually composed similarly. While three quarters on the left side are dominated by the animal, the right margin is dominated by water. Yet many greenish or brownish images with a brindled texture have been retrieved. The impact of colours should have been higher.

\section{Query 6(a)}

In this case the Wavelet can play out its strengths. Every single image has been found, even if the lighting and the waves on the water changed. The feature vector seems to be very robust against these changes. The Histogram also performed very well, but it missed out the image with the strongest change in lighting. The two other features seem to have stumbled over the lack of difference in the similarity. Both ranked other images way too high. This query is not very specific about the bird in the center. Instead, most of the image is featureless background showing a water surface. Taking the Spatial Histogram, about 40 results in the first 50 hits actually show birds surrounded by water.

\section{Query 6(c)}

Here three of the four feature vectors were obviously suitable. They are very robust against the slight changes in the images. Especially the Histogram feature was able to find all relevant images. The only feature with not a single hit is again the Spatial Histogram. Most of the retrieved results contain much blue and a darker, brownish central part. Again the ranking is blinded by the other possible matches. The modifications in the image composition seem to be too strong to be caught.

\section{Query 6(d)}

At this point the retrieval gets wrong with all four features even if each one is able to find some of the related images. Each feature actually finds different images of the targeted ones. For the Spatial Histogram the shifting perspective remains the main problem. In general the image colours from many images are similar.

\section{Query 6(e)}

The bird sitting in the green grass is a very captivating query. The image is dominated by the grass and with a closer look the grass pattern of the query image is indeed different from the other ones. Taking this into account it is not very surprising that features focusing on the spatial information fail. In this case the Wavelet feature is the only one with no results. The RGB Mean obviously benefits from the fact that most images contain different shades of green. If another image of the series is selected for query, all features are capable of retrieving most images correctly.

\section{Query 6(f)}

This series again contains two differently illuminated sub series. In the query one of the lighter set is used. Under those conditions the most detailed features Spatial Histogram and Wavelet are at least able to pick the correct sub series. The two simpler features fail because they rank too many wrong images too high. In this case a feature robust to the change of lighting seems to be appropriate.

\section{Query 6(g)}

The last query is indeed very challenging. None of the feature plug-ins was able to find even a single match of the series (Fig. 7). In the human perception these three images are actually quite similar, but the implemented features obviously did not capture the relevant information to achieve good ranking results.

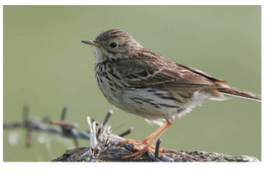

(a)

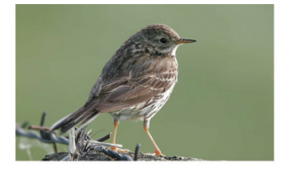

(b)

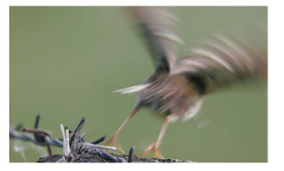

(c)
Fig. (7). Meadow pipit series 6 .

\section{Conclusion}

The analysis of the different features acknowledges that each feature vector has certain strengths and weaknesses.

The remarkably good performance of the RGB Mean in special cases is surprising. At least it is very robust against several changes in the image composition as long as the colour does not change very much. Of course this feature gets very weak as soon as there are other images with similar colours.

The lighting of the scene has a visible impact on the retrieval. Under these circumstances a feature with a high robustness against it is required. It should be based on shapes or maybe also wavelets without colour information. Conceivably also a region based approach would be beneficial in many cases.

\section{Multi Feature Language}

Testing the multi feature ability revealed many advantages compared to the single feature search. Nevertheless there is still much work to do.

Using the query language with boolean clauses sometimes helps to improve the result. Especially searching for the third bird series (Fig. (6d)) was much more efficient by using three SHOULD clauses with different features. Of course the following optimisation effort by adding several NOT clauses does not seem to be very realistic in daily use, but it showed that a directed filtering may indeed help. It needs to be pointed out that it is advisable to use different features for NOT clauses to avoid that correct hits are removed. 
Another issue when combining two features is the discrepancy of similarities. The normalisation to a the range from 0.0 to 1.0 is only part of the solution. In addition the calculation needs to be calibrated to be efficient. Some features return many results with a similarity above 0.99 (especially RGB Mean), while others have a much lower similarity even for images from the same set. The Wavelet implementation often returned similarities of about 0.75 . Merging the two subsets is very inefficient because the less distinctive features are dominant. Altering the boost for single terms can be used as a workaround to reduce the problem.

The effort of constructing a well performing query may be too high in many retrieval scenarios if the user only wants to find a certain image. In other scenarios, the optimised query can be seen as some kind of classificator for a certain type of images.

\section{Retrieval Hints}

Using the advanced capabilities of the query language requires deeper understanding and some experience with the underlying ranking system. For this prototype some default techniques should be used.

If a fuzzy retrieval is performed, the clauses in a boolean query should be used normally, i.e. they are best joined by SHOULD. This ensures that no relevant document is cut away from the result set.

Having a more specific query with a high separability between hit and miss, the MUST clause is helpful to efficiently restrict search space.

Finally the MUST NOT clause needs to be used carefully. It is useful for removing a certain image series completely from the result set, if it is annotated and the wanted results are not. Using it for a fuzzy search term may be dangerous, because all of the documents contained in the sub result are removed. In this case the size of the MUST NOT clause should be restricted by appropriate parameters.

\section{Recommended Tools}

Optimising queries is already possible with the current user interface but it is a tedious task. Some additional tools seem to be beneficial.

It should be possible to mark all desired images. Then the engine may check whether single documents are missing when performing a refined query. Especially when adding MUST and MUST NOT terms, a warning message could be generated.

Especially for MUST NOT clauses it might be helpful to see a preview which documents are going to be filtered out. This helps to tune the restriction parameters and to control the size of the filtered out documents.

In a productive environment the similarity could be spread by a power-law function analogous to the gamma correction known from image processing. Based on a set of reference images the parameters could be tuned to achieve a sound calibration.

\section{User Survey}

In general the testers behaved as expected and solved the tasks. Additional knowledge of certain image content (e.g. bird names) sometimes sped up the retrieval drastically. Where no or insufficient annotation was available, the search took much longer.

Some of the main critics were actually intentionally built into the repository. Especially the demand for better keywords clearly shows that a sound annotation is a very important factor for retrieval systems. Extending the current keyword search to a more powerful semantic environment like topic maps could boost the quality. An internal substitution mechanism of very specific words to more general ones could be the first step.

What needs to be done as soon as possible is to create a simple user interface where queries can be easily composed and images can be uploaded. Also an integrated drawing tool seems to be beneficial. Especially the features which are difficult to understand could profit by a stronger integration and by hiding the complexity.

Further the testers had almost no problems in understanding the query language and the boolean operators. During the first testing sessions a small bug in the ranking system caused strange effects. Fortunately a simple workaround in the query was enough to avoid serious problems and the bug could be fixed soon. The three different operators MUST, SHOULD and MUSTNOT were accepted by all testers.

All testers are quite professional computer users and have some experience in searching. But no one had experienced a CBIR system before and the user interface was very basic. Keeping that in mind, this is still a remarkable success for the query language.

\section{Addendum}

At the time of the main survey, the user interface only consisted of a simple HTML web page. Queries had to be typed manually into a single text field. Many problems were caused by syntax errors. In the beginning, it usually took a couple of attempts to formulate a valid query. After a while, they got accustomed to the language and were able to create more complex ones. This quick learning was surely affected by the fact that most testers were used to programming languages.

In the current prototype, a visual query composer has been added. For evaluation purposes, a testing person with no programming experience performed the same survey with the new interface. Due to the fact, that there was only a single tester, this second survey was absolutely nonrepresentative. Nonetheless, it indicated, that the visual composer can be a helpful tool. The tester clearly preferred the visual composer to the plain text field but still understood the language itself. The new functionality to specify an external query image was considered helpful. In one case, the drawn query (Fig. (8b)) was good enough to retrieve the requested image (Fig. (8a)) at the first position.

\section{CONCLUSION}

\section{Achievements}

The proposed query language has a simple structure and is very similar to a full text search engine while also allowing fuzzy terms. Further it is easily extensible and allows arbitrary constructs for individual features. Complex queries 
are possible but not necessary, giving experts the chance to fine tune all parameters as required. Normal users could either enter simple queries or generate them with a graphical user interface.

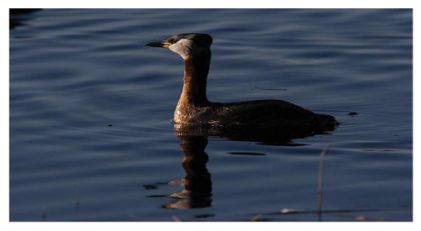

(a)

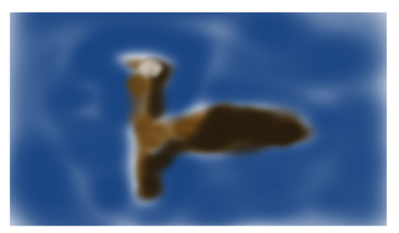

(b)
Fig. (8). Query-by-example.

One interesting feature of boolean queries is the ability to reduce the search space. Setting the occurrences of the clauses restrictively, it provides a way to cut the retrieval time drastically, even if the CBIR terms require a linear scan.

Further the language can be easily mapped to machine readable formats like objects or XML.

\section{Problems Remaining}

Providing a basic parser like JSON only simplifies the low-level query information. To support higher abstractions it is necessary to fully understand the feature itself, which is impossible for a generic language. For this reason, keeping the language simple is the task of the feature developers. They need to design appropriate sub languages which contain all feature specific information and remain as readable as possible.

Another issue is the naming of feature vector based fields. Currently the prototype compares each field name in the query with the list of available feature plug-in identifiers. If the field name does not match a feature identifier, the term is handled by the underlying Lucene engine, executing a "classical" full text search on the field. Otherwise the term is forwarded to the corresponding feature plug-in. Having overlapping feature identifiers, basic search fields could be hidden. It is necessary to formulate naming conventions like reserved words or a prefix for each feature identifier.

\section{Future Work}

Unlike FOQL/SQL the language does not support user defined sorting like ORDER BY but sorts results by an overall similarity. It is to decide whether this extension is relevant for retrieval issues or not.

Depending on the combining functions and feature vectors used, query processing can be sped up drastically. A heuristic approach to query optimizing has been evaluated by Ramakrishna (Ramakrishna ADC 2002) [15].

The currently implemented indexing structures for the plug-ins are merely a proof-of-concept. A set of generic indexing structures (such as for multidimensional vectors) is planned to support standard types of features directly.

Another crucial topic is the merging strategies. Especially for iterative search, additional work needs to be done. Until now, the engine always returns the optimal result by checking all features and keeping the whole result in the memory. An approach to get page-wise additional results as requested is planned.

The query language proposed in this article does not yet support query-by-example directly. This requires to encode pixel images in a string, which may be done by mime encoding.

The support of high-level concepts is not realized yet. This could be a feature of the language itself by introducing constructs like define in FOQL which substitute certain terms by a pre-defined low level term. Alternatively the retrieval engine itself could be extended by high-level plug-ins which map semantics to predefined low level requests. Developing such feature plug-ins is a very complex task. A lot of testing is required to capture meaningful feature vectors information which represents semantics.

\section{ACKNOWLEDGEMENTS}

A major part of the testing repository images were contributed by external persons. Many thanks to Prof. Dr. Jörg Raasch from the Hamburg University of Applied Sciences for his large and nicely annotated collection. Also thanks to Benjamin Striepke for his contribution of many images.

Finally, I want to thank all testers involved in the usability study and gave much useful feedback.

\section{REFERENCES}

[1] J. Eakins and M. Graham, "Content-based Image Retrieval. A Report to the JISC Technology Applications Programme," University of Northumbria at Newcastle, Tech. Rep., Jan. 1999. [Online]. Available: http://www.jisc.ac.uk/uploaded documents/ jtap-039.doc

[2] M. Renz and W. Renz, "Neue Verfahren im Bildretrieval. Perspektiven f ur die Anwendung," in Proceedings der 22. Online-Tagung der DGI, R. Schmidt, Ed., May 2000, pp. 102-128.

[3] Y. Liu, D. Zhang, G. Lu, and W.-Y. Ma, "A survey of contentbased image retrieval with high-level semantics," Pattern Recognition, vol. 40, pp. 262-282, 2007.

[4] P. Clough, H. Müller, T. Deselaers, M. Grubinger, T. M. Lehmann, J. Jensen, and W. Hersh, Accessing Multilingual Information Repositories. Springer, 2006, vol. 4022/2006, ch. The CLEF 2005 Cross-Language Image Retrieval Track, pp. 535-557.

[5] P. Clough, M. Grubinger, T. Deselaers, A. Han-bury, and H. M* uller, Evaluation of Multilingual and Multi-modal Information Retrieval. Springer, 2007, vol. 4730/2007, ch. Overview of the ImageCLEF 2006 Photographic Retrieval and Object Annotation Tasks, pp. 579-594.

[6] R.Fagin, "Combining fuzzy information from multiple systems (extended abstract)," in PODS '96: Proceedings of the fifteenth ACM SIGACTSIGMOD-SIGART symposium on Principles of database systems. New York, NY, USA: ACM, 1996, pp. 216-226.

[7] Apache Software Foundation, "Apache Lucene," 2006. [Online]. Available: http://lucene.apache.org/

[8] R. G. G. Cattell, "ODMG-93: a standard for object-oriented DBMSs," in SIGMOD '94: Proceedings of the 1994 ACM SIGMOD international conference on Management of data. New York, NY, USA: ACM, 1994, p. 480.

[9] A. M. Alashqur, S. Y. W. Su, and H. Lam, "OQL: a query language for manipulating object-oriented databases," in VLDB '89: Proceedings of the 15th international conference on Very large data bases. San Francisco, CA, USA: Morgan Kaufmann Publishers Inc., 1989, pp. 433-442.

[10] S. Nepal and M. Ramakrishna, "Query Processing Issues in Image(Multimedia) Databases," International Conference on Data Engineering, 1999, pp. 22-29.

[11] C. Town and D. Sinclair, "Ontological query language for content based image retrieval," in Content-Based Access of Image and Video Libraries, 2001. (CBAIVL 2001). IEEE Workshop on, 14 Dec. 2001, pp. 75-80. 
[12] C. Town and D.Sinclair, "Language-based querying of image collections on the basis of an extensible ontology," Image and Vision Computing, vol. 22, pp. 251-267, 2004.

[13] J. Martinez, R. Koenen, and F. Pereira, "MPEG-7: The Generic Multimedia Content Description Standard, Part 1," IEEE MultiMedia, vol. 09, no. 2, pp. 78-87, 2002.

[14] D. Riecks, "'IPTC Core" Schema for XMP -Version 1.0," International Press Telecommunications Council, Tech. Rep., 2005.

[15] M. V. Ramakrishna, S. Nepal, and P. K. Srivastava, "A heuristic for combining fuzzy results in multimedia databases," in $A D C$ ' 02 : Proceedings of the 13th Australasian database conference. Darlinghurst, Australia, Australia: Australian Computer Society, Inc., 2002, pp. 141-144.

[16] L. A. Zadeh, Fuzzy sets. River Edge, NJ, USA: World Scientific Publishing Co., Inc., 1996.

[17] R. P. Pein and Z. Lu, "A Flexible Image Retrieval Framework," in International Conference on Computational Science (3), ser. Lecture Notes in Computer Science, Y. Shi, G. D. van Albada, J. Dongarra, and P. M. A. Sloot, Eds., vol. 4489. Springer, may 2007, pp. 754-761.

[18] F. A. Al-Omari and M. A. Al-Jarrah, "Query by image and video content: a colored-based stochastic model approach," Data Knowl. Eng., vol. 52, no. 3, pp. 313-332, 2005.
[19] C. E. Jacobs, A. Finkelstein, and D. H. Salesin, "Fast Multiresolution Image Querying," Computer Graphics, vol. 29, no. Annual Conference Series, pp. 277-286, 1995. [Online]. Available: citeseer.ist. psu.edu/jacobs95fast.html

[20] R.P. Pein and Z. Lu, "Content Based Image Retrieval by Combining Features and Query-By-Sketch." in International Conference on Information \& Knowledge Engineering (IKE), H. R. Arabnia and R. R. Hashemi, Eds. Las Vegas, USA: CSREA Press, Jun. 2006, pp. 49-55.

[21] R. P. Pein, "Hot-Pluggable Multi-Feature Search Engine," Master's thesis, Hamburg University of Applied Sciences, 2008.

[22] R. P. Pein, M. Amador, J. Lu, and W. Renz, "Using CBIR and Semantics in 3D-Model Retrieval," in Computer and Information Technology, 2008. CIT 2008. 8th IEEE International Conference on, Jul. 2008, pp. 173-178.

[23] R. Fergus, P. Perona, and A. Zisserman, "Object class recognition by unsupervised scale-invariant learning," in Proceedings of the IEEE Conference on Computer Vision and Pattern Recognition, vol. 2, Madison, Wisconsin, June 2003, pp. 264-271.

(C) Pein et al.; Licensee Bentham Open.

This is an open access article licensed under the terms of the Creative Commons Attribution Non-Commercial License (http://creativecommons.org/licenses/by-nc/3.0/) which permits unrestricted, non-commercial use, distribution and reproduction in any medium, provided the work is properly cited. 